\title{
Multimodal and In-Situ Chemical Imaging of Critical Surfaces and Interfaces in Li Batteries
}

\section{Chong-Min Wang, Zihua Zhu, Mark H. Engelhard, Arun Devaraj, and Donald R. Baer*}

Environmental Molecular Sciences Laboratory, Pacific Northwest National Laboratory, 902 Battelle Boulevard, Richland, WA 99352

don.baer@pnnl.gov

\begin{abstract}
This article describes ways that a range of microscopy, spectroscopy, and spectrometry tools are being used to address important challenges associated with energy storage science and technology, in particular the development of advanced batteries for transportation, consumer use, and renewable storage. In this article in-situ transmission electron microscopy, in-situ secondary ion mass spectrometry, and $\mathrm{x}$-ray photoelectron spectrometry (XPS) have been used to examine the formation and properties of the solid-electrolyte interphase (SEI) layer. Also transmission electron microscopy (TEM), atom probe tomography (APT), and high spatial resolution SIMS (nanoSIMS) have been used to optimize the structure and processing of a lithium transition metal oxide cathode.
\end{abstract}

\section{Introduction}

The development of longer-lasting, higher-capacity, lighter-weight, and/or lower-cost batteries relevant to consumer electronics, electric transportation, and sustainable energy storage presents a variety of scientific and technological challenges [1-2]. Many of these challenges concern the performance and stability of electrodes, the electrolyte that connects them, and the solid-electrolyte interphase (SEI) layer that forms between them [3-5]. Enhancement of the properties of current-generation Li-ion batteries and developing next-generation batteries (beyond lithium) is the subject of considerable private and public research.

An increasing variety of analytical tools is used to address the complex issues associated with electrode design, performance, and aging. These tools facilitate research focused on understanding the formation and operating properties of the SEI layer. Significant advances are being achieved through the use of tools that can examine batteries during operation (in situ or operando), although there remain roles for conventional and $e x$-situ methods that can provide complementary information not yet available using

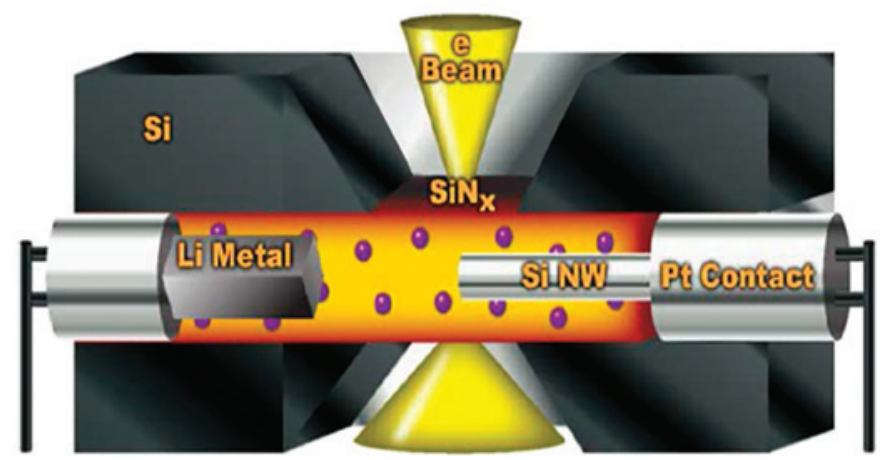

Figure 1: Schematic drawing illustrating the fundamental concept of a closedcell in-situ STEM probe for the study of Li-ion batteries. Reproduced with permission from [11]. Copyright 2013, American Chemical Society. in-situ methods. Because of the environmental sensitivity of battery material, both in-situ and ex-situ analysis methods benefit from specialized probes and sample handling adapted to controlled environments.

In this article we focus attention on two related but distinct battery issues that demonstrate how a range of tools, in-situ and $e x$-situ, are used in battery research. The first issue (A) concerns SEI layers: how they form, their properties, and how they change as a function of time or charge/discharge cycles. The work we describe involves analysis of $\mathrm{Li}$-ion and Li-air batteries. The second topic (B) focuses on the development and optimization of lithium-transition-metal-oxide-based cathode materials.

\section{Materials and Methods}

Analytical tools are continuously improving in sensitivity as well as spatial and temporal resolution, and they are being extended to operation in increasingly relevant environments (in-situ and operando studies) [6-8]. However, it is increasingly recognized that a single analytical tool, even if highly versatile, can provide only limited information. Most often full understanding of a material's behavior requires complementary information captured with multiple types of instrumentation, sometimes with significantly different spatial and temporal resolution. Consequently, the significant challenge for the scientific community is to find ways to apply multiple types of instrumentation to analyze a specific site or region of the sample without damage to the desired information. The techniques used in this article are in-situ and ex-situ scanning transmission electron microscopy (STEM), energy-dispersive X-ray spectroscopy (EDS), x-ray photoelectron spectrometry (XPS), in-situ time-of-flight secondary ion mass spectrometry (ToF-SIMS), atom probe tomography (APT), and high spatial resolution SIMS (NanoSIMS). The latter three techniques can provide isotope distributions and trace element detection in addition to element identification and quantification.

\section{Results}

(A) Structure and Chemical Composition of the Solid-Electrolyte Interphase (SEI).

In-situ TEM study of SEI layer formed on Pt in model Li-ion battery The electrodes of alkali batteries of various types (Li-ion, Li-air, Li-S, Na-ion, and Mg-ion) undergo a variety of structural changes during charge and discharge cycles. The phases that form in the SEI layer may capture working ions as well as form dead layers or diffusion barriers, all of which can inhibit cycling and degrade battery performance. A wide variety of electrode designs have been 


\section{June $5-10,2016$}

\section{LEHIGH}

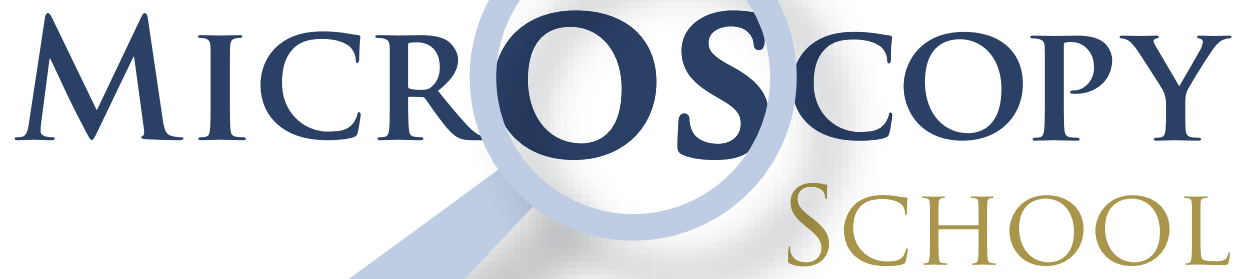

Lehigh University, Bethlehem, PA USA

\section{MAIN COURSES}

SCANNING ELECTRON MICROSCOPY AND X-RAY MICROANALYSIS

June 6-10

\section{SPECIALIZED COURSES}

FOCUSED ION BEAM (FIB): Instrumentation and Applications June 6-10

PROBLEM SOLVING:

Interpretation and Analysis of SEM/EDS/EBSD Data June 6-10
INTRODUCTION TO SEM AND EDS FOR THE NEW OPERATOR June 5
QUANTITATIVE X-RAY

MICROANALYSIS:

Problem Solving using EDS and WDS Techniques June 6-10

SCANNING TRANSMISSION ELECTRON MICROSCOPY:

From Fundamentals to Advanced Applications June 6-10

For more information, contact:

Sharon Coe | 610.758.5133 | sharon.coe@lehigh.edu 


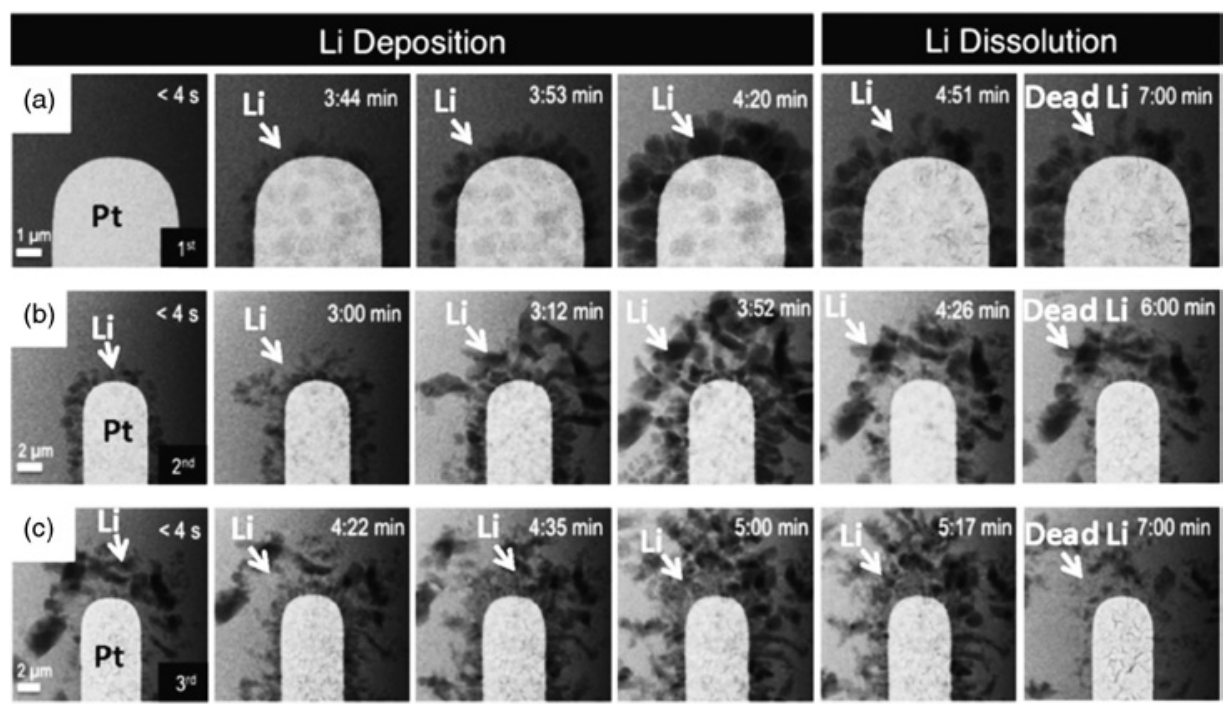

Figure 2: High-angle annular dark-field (HAADF) images of Li deposition and dissolution at the the Pt working electrode using $\mathrm{LiPF}_{6} / \mathrm{PC}$ electrolyte during the (a) first, (b) second, and (c) third charge/discharge cycles of the operando cell. The formation of the "dead Li" on the electrode can be seen in the images at the end of the Li dissolution cycle (the far right column of images), thereby demonstrating the degree of irreversibility associated with the battery cycling. Reproduced with permission from [12]. Copyright 2015, American Chemical Society.

(a)

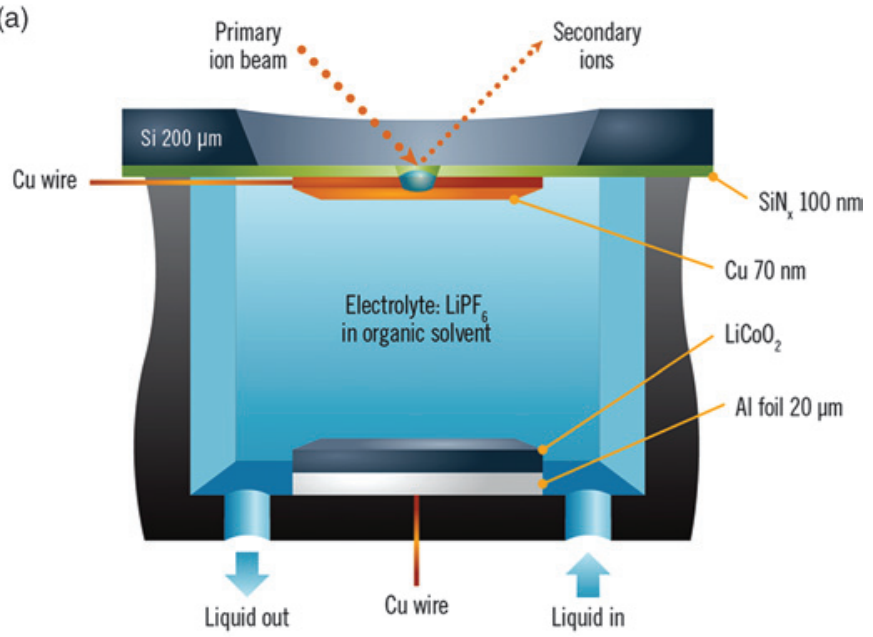

(b)

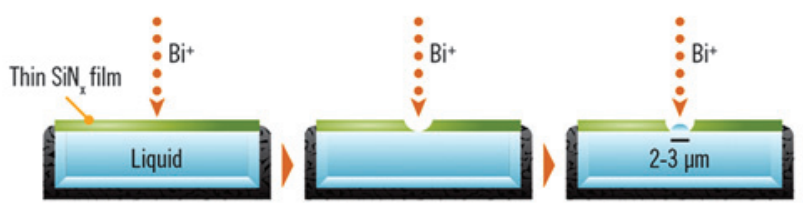

(c)

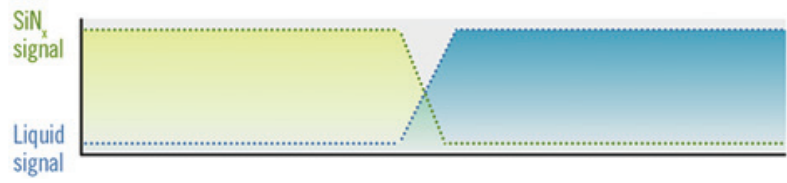

Figure 3: Conceptual drawing of a battery that can be examined in a SIMS system. (a) The assembled battery showing the thin $\mathrm{SiN}_{\mathrm{x}}$ layer upon which a $\mathrm{Cu}$ anode is deposited. The $\mathrm{LiCoO}_{2}$ layer is the cathode. (b) Schematic drawing showing the principle of in-situ liquid SIMS for which $\mathrm{Bi}^{+}$ions were used as the primary ion for sputtering toward the solid-liquid interface. (c) SIMS signal as a functional of sputtering time (or depth) as the primary ion beam sputters through the thin $\mathrm{SiN}_{\mathrm{x}}$ film. When the primary ion beam just sputters through the $\mathrm{SiN}_{\mathrm{x}}$ film, the elemental, isotopic, and molecular information at the solid-liquid interface can be detected. conceptualized and synthesized in the effort to minimize both electrode changes and growth of deleterious SEI layers. In-situ probes, that are in essence model batteries, provide a way to actually observe how the material behaves during charge and discharge cycles. In-situ tools based on TEMs and SEMs employing open cells $[9,10]$ or closed cells $[7,11]$ have developed rapidly in the last five years. Figure 1 shows a schematic drawing to illustrate the general concept of the closed cell in-situ TEM "battery," enabling the observation of electrode evolution in a model battery with realistic liquid electrolytes [11]. As an example, this in-situ TEM cell was used to observe lithium dendrite growth in STEM mode. The deposition of $\mathrm{Li}$ on a Pt electrode in $\mathrm{LiPF}_{6} / \mathrm{PC}$ electrolyte is shown in Figure 2 [12]. With reversal of the voltage, the Li gets stripped off. Figure 2 provides direct morphological information of the deposited lithium related species and simultaneously reveals that the deposition and stripping off of the $\mathrm{Li}$ is not fully reversible. The non-reversibility of the $\mathrm{Li}$ deposition provides important information regarding the loss of accessible Li during battery operation. Although highly informative, a significant limitation of in-situ TEM/STEM measurements is the difficulty of obtaining chemical information of the deposited lithium-related species. When operating in a transmission liquid cell, the image and X-ray spectroscopic information obtained will inevitably include signals from the liquid electrolyte as well as the region of interest. Even electron energy loss spectroscopy (EELS) from in-situ liquid cells will yield a chemical composition that integrates multiple regions of the model battery. Deconvolution of the molecular structure in the SEI layer would be very difficult, if not impossible. Therefore, it is important to identify ways to obtain complementary information, especially about the molecular processes occurring at electrode surfaces.

In-situ SIMS of electrolyte-electrode interactions and SEI development. During charge and discharge cycles of a battery, migration and reaction of mobile ionic species in the electrolyte cause structural and chemical modification of both electrode surfaces and the electrolyte. These chemical modifications are not well understood, and it is highly challenging to directly probe the liquid electrolyte-solid interface. A newly designed in-situ cell (Figure 3a) makes it possible to use SIMS to directly observe the structural evolution on the electrode and within the liquid electrolyte for a model lithium ion battery under dynamic operating conditions [13]. Similar to the in-situ TEM battery cell, the model battery used for SIMS measurements contains a thin 
(a)

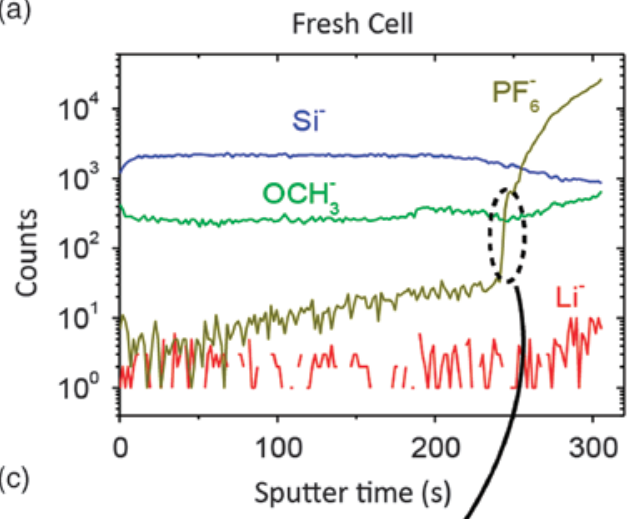

(b)

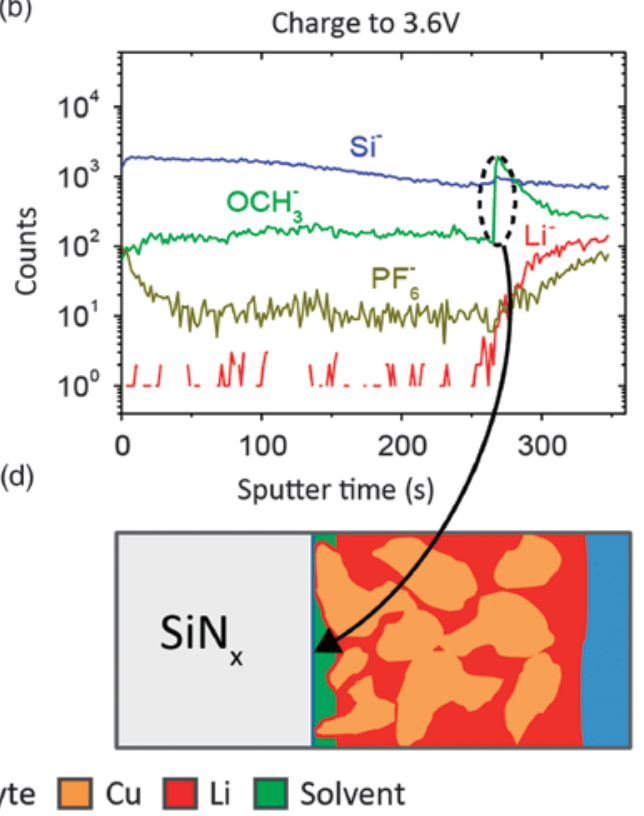

Figure 4: ToF-SIMS depth profiles of several representative negative ion species. (a) In a fresh cell, the $\mathrm{PF}_{6}{ }^{-}$signal dramatically increases after punching through the $\mathrm{SiN}_{\mathrm{x}}$ film, indicating that electrolyte can diffuse to the $\mathrm{SiN}$ - $\mathrm{Cu}$ interface, which is supported by the SEM observation that the $\mathrm{Cu}$ film is porous. This situation is schematically illustrated in (c). (b) After charging, a jump of solvation signals $\left(\mathrm{OCH}_{3}{ }^{-}\right.$as an example) appears at the $\mathrm{SiN}-\mathrm{Cu}$ interface, but the $\mathrm{PF}_{6}{ }^{-}$signal greatly decreases, indicating a solvent layer depleted in LiPF 6 forms at the interface. Also, the Li- signal significantly increases after charging, suggesting that metal Li forms after charging. This situation is schematically illustrated in (d).

$\mathrm{SiN}_{\mathrm{x}}$ layer. In this case a copper layer is deposited onto the window to serve as the battery anode. In contrast to the thin window used for in-situ TEM, which needs to remain intact for the duration of the experiment, for SIMS an ion beam is used to create a $2 \mu \mathrm{m}$ hole large enough so that SIMS spectra can be collected, but small enough for capillary forces to minimize the loss of the electrolyte into the vacuum. Figure $3 \mathrm{~b}$ shows the process of using an ion beam to create a hole in the $\mathrm{SiN}_{\mathrm{x}}$ window to allow SIMS spectra to be collected

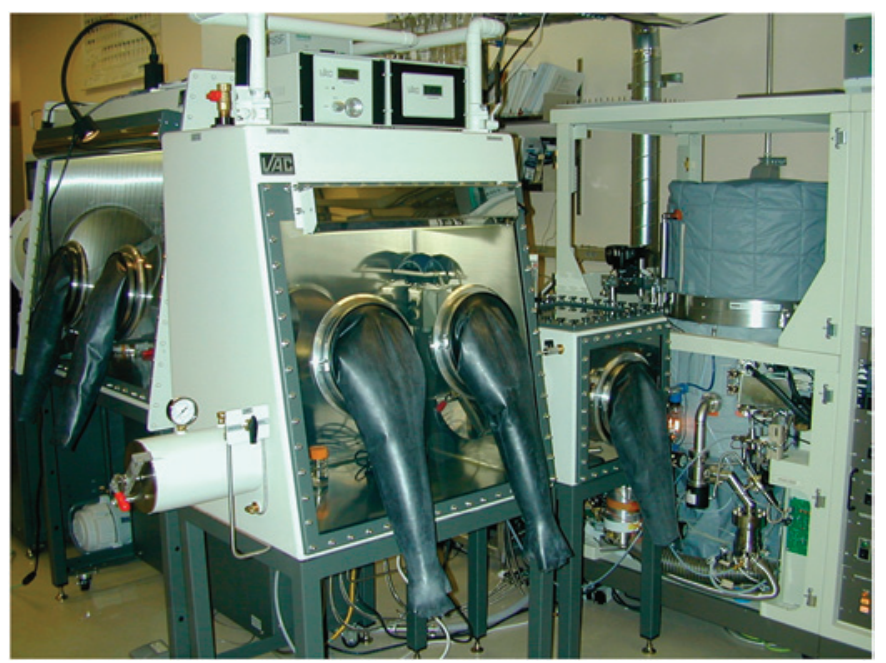

Figure 5: Glove box for anaerobic handling of battery components during and after disassembly in controlled environments in preparation for XPS measurements. liquid electrolyte. Evidence for this includes the significant jump of the $\mathrm{PF}_{6}{ }^{-}\left(\mathrm{m} / \mathrm{z}\right.$ 145) peak when the $\mathrm{SiN}_{\mathrm{X}}$ layer is penetrated. The presence of the liquid layer makes it impossible to sputter through the porous $\mathrm{Cu}$ electrode as evidenced by the $\mathrm{Cu}^{+}$depth profile (not shown in Figure 4) [13]. Upon charging, Li metal was found deposited on $\mathrm{Cu}$ electrode, and condensation of solvent molecules was observed around the electrode. Chemically, this layer of solvent condensate is depleted of both $\mathrm{Li}^{+}$and $\mathrm{PF}_{6}{ }^{-}$ions, forms an ionic insulating layer, and therefore contributes to the over-potential of the cell. Evidence for these effects include: the increase in the $\mathrm{Li}^{-}$(peak at $\mathrm{m} / \mathrm{z}=7$ ) at the interface in comparison to the fresh battery, a significant drop of the $\mathrm{PF}_{6}{ }^{-}(\mathrm{m} / \mathrm{z}=145)$ at the interface in comparison to the fresh battery, and the dramatic jump of the $\mathrm{OCH}_{3}{ }^{-}(\mathrm{m} / \mathrm{z}=31)$ when the $\mathrm{SiN}_{\mathrm{x}}$ layer is penetrated.

During charge and discharge cycles, insoluble $\mathrm{Li}$ salts gradually form and accumulate on the $\mathrm{Cu}$ electrode surface [13] (similar to the TEM observations above). The in-situ liquid SIMS measurements provide a new way to directly obtain molecular information at the electrode surface. This information complements that available by electron microscopy and has the potential to significantly impact other research work dealing with liquid-solid interfaces under dynamic operating conditions.

XPS study of SEI development as a function of charge. While there are efforts to mitigate the limitations of Li-ion batteries, there is also an aggressive search for other types of cost-effective and safe rechargeable batteries with higher-energy densities and 
(a)

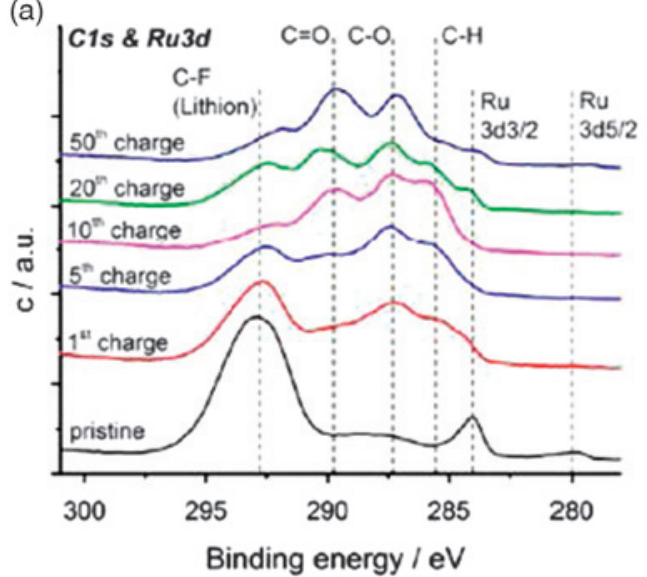

(c)

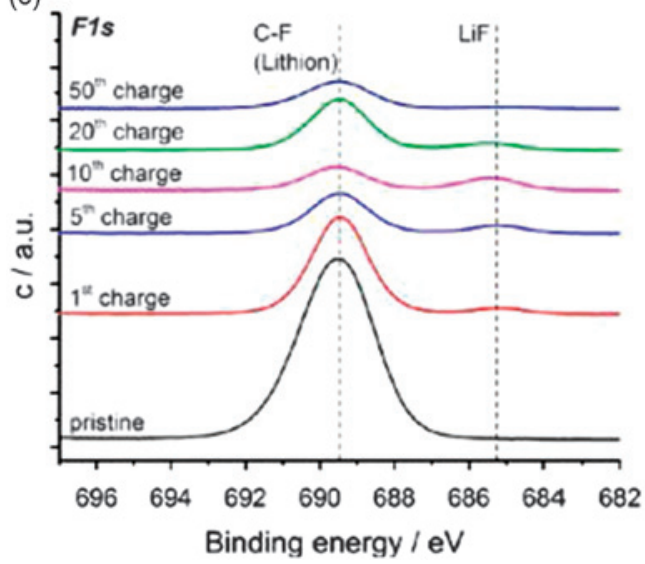

(b)

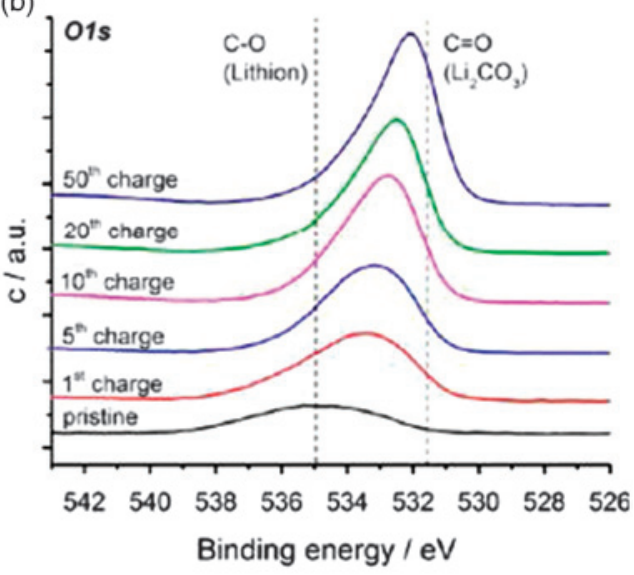

(d)

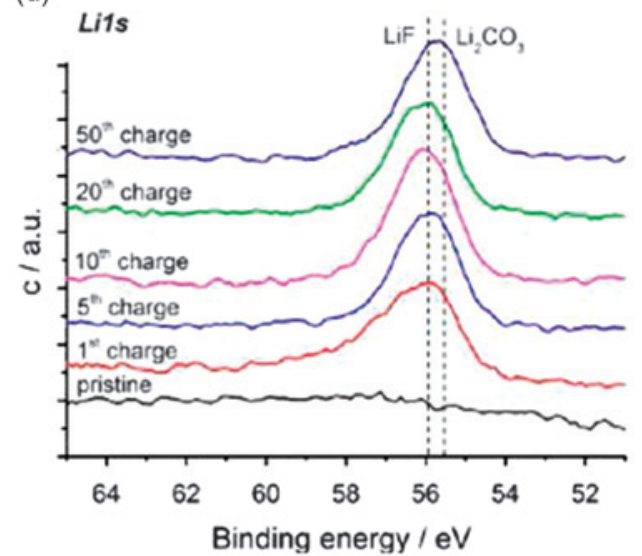

Figure 6: XPS results for pristine and charged CNTs/Ru electrodes scanned in (a) C 1s and Ru 3d, (b) O 1s, (c) F 1s, and (d) Li 1s regions. Changes in these spectra after charge and discharge cycles highlight the decomposition of the electrolyte and breakdown of CNTs. Reproduced with permission from [14]. Copyright 2014, American Chemical Society.

longer lifetimes. Among all sustainable rechargeable Li-batteries, $\mathrm{Li}-\mathrm{O}_{2}$ and $\mathrm{Li}-\mathrm{S}$ batteries appear most promising with very high specific energy densities.

XPS is increasingly used to analyze the components and compositions of surface layers ( $0.1 \mathrm{~nm}$ to a few $\mathrm{nm}$ ) on battery electrodes. In order to extract materials exposed to relevant conditions, battery cells were opened in a controlled atmosphere afterbeing cycled to the desiredpotentials. Aglovebox (Figure5), or other controlled-atmosphere transfer system, is required to avoid or minimize oxidation and contamination of the exposed electrodes.

As one example, XPS has been used to analyze the changes in the chemistry of $\mathrm{RuO}_{2}$-incorporated carbon nanotube (CNT) air electrodes after long-term operation of $\mathrm{Li}-\mathrm{O}_{2}$ batteries under full discharge/charge conditions in a glyme-based electrolyte (Figure 6) [14]. Spectra including the $\mathrm{Ru} 3 \mathrm{~d}_{5 / 2}, \mathrm{C} 1 \mathrm{~s}, \mathrm{O} 1 \mathrm{~s}, \mathrm{~F} 1 \mathrm{~s}$, and Li 1s photoelectron peaks were examined as a function of charging cycles. The evolution of the spectra with the cycling of the battery as shown in Figure 6 clearly indicates the chemical evolution of the surface of the electrode, signifying the formation of side products generated from decomposition of the electrolyte, an increase in oxidized carbon $(\mathrm{C}=\mathrm{O}$-containing species), and the breakdown of the
CNTs. The surface sensitivity of XPS results provided important information about the nature of the interface and electrode changes during cycling that impact electrode stability and battery lifetime.

XPS measurements under controlled atmospheres as a function of time and at different potentials have been useful during battery development, testing, and failure analysis. However, even "routine" XPS measurements of electrodes and battery materials as they are assembled have been helpful in identifying "unintended" surface species (too small to be routinely identified by many other measurements) that occasionally appear during routine production and handling of materials. When XPS was conducted routinely, these problems were identified before battery testing experiments. Often XPS can help identify causes of experimental failure or lack of reproducibility.

(B) Effects of Elemental Distributions on Battery Performance.

$\mathrm{Ni}$ segregation in individual $\mathrm{Li}_{1.2} \mathrm{Ni}_{0.2} \mathrm{Mn}_{0.6} \mathrm{O}_{2} \quad$ cathode particles. One approach to improve battery performance has been the incorporation of multivalence transition-metal ions into metal oxide cathodes [15]. One system getting particular attention has been lithium-nickel-manganese-oxide cathodes for which the addition of nickel has been found to significantly improve battery performance. The biggest challenge limiting use of these layer-structured cathodes in lithium-ion batteries has been capacity and voltage fading. The power rate of a battery strongly depends on the lithium ion drift speed in the cathode. The classic wisdom regarding layerstructured materials is that lithium ion travels swiftly between the layers, moving out/in of the cathode during the charge/ discharge cycles. The STEM-EDS analysis of as-synthesized electrode material (Figures $7 \mathrm{a}-7 \mathrm{f}$ ) provided the unexpected discovery of a thermodynamically driven, yet kinetically controlled, segregation of $\mathrm{Ni}$ to surface facets of fresh electrode particles. This segregation served as a barrier to lithium diffusion near the surface of a particle $[3,16]$. Therefore, the transition-metal dopant that helps provide high capacity and/ or high voltage can be located in a "wrong" location and may inhibit lithium diffusion, limiting battery performance [17].

Although STEM-EDS can readily examine the relatively heavy elements, it is difficult to determine the location of $\mathrm{Li}$ or quantitatively provide the composition of different 
(a)

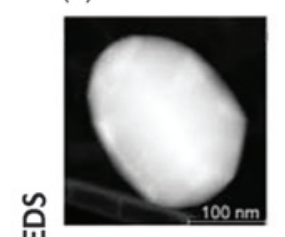

$+(d)$

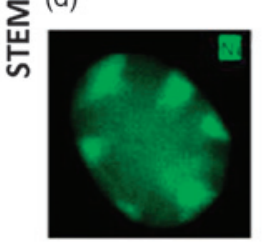

(b)

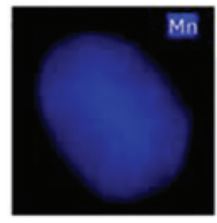

(e)

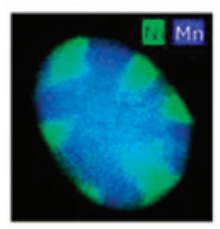

(c)

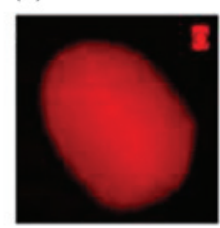

(f) $\mathrm{Mn} /(\mathrm{Mn}+\mathrm{Ni})$
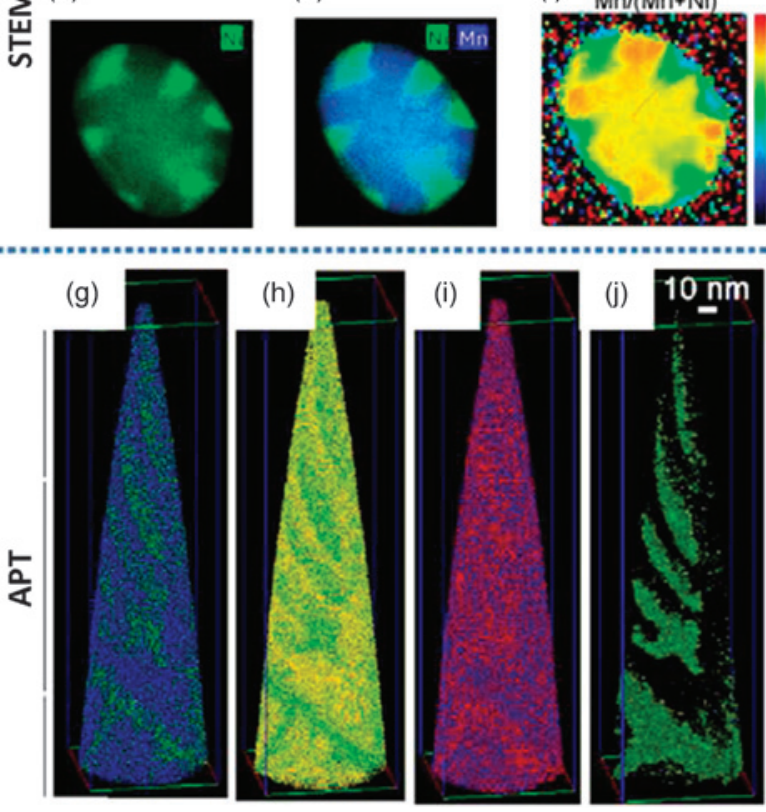

(g)
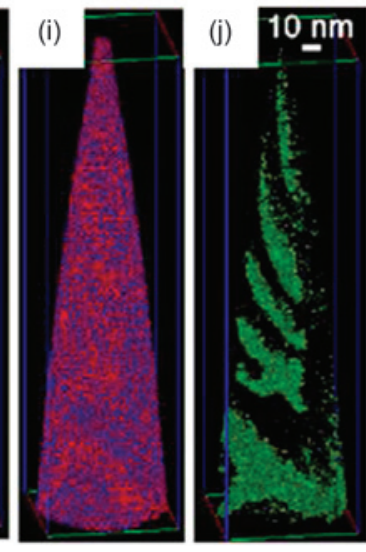

Figure 7: Comparison of STEM EDS mapping with APT results of the layered $\mathrm{Li}_{1.2} \mathrm{Ni}_{0.2} \mathrm{Mn}_{0.6} \mathrm{O}_{2}$. (a) STEM image and EDS maps of (b) $\mathrm{Mn}$ (blue), (c) $\mathrm{O}$ (red), (d) $\mathrm{Ni}$ (green), (e) $\mathrm{Ni}$ and $\mathrm{Mn}$ composite map, and (f) ratio of $\mathrm{Mn} /(\mathrm{Mn}+\mathrm{Ni})$. The intensity key, from bottom to top, represents increasing ratio. Because this is a ratio map, the low count outside of the particle gives large errors in dividing and causes the reddish background. APT: two-element composite maps of APT reconstructions are shown from (g) to (j) where each dot corresponds to an atom in the reconstruction. (g) $\mathrm{Mn}$ (blue) and Ni (green), (h) Li (yellow) and Ni (green), (i) $\mathrm{O}$ (red) and $\mathrm{Mn}$ (blue), and (j) 13 at\% $\mathrm{Ni}$ isocomposition surface highlighting the $\mathrm{Ni}$-rich regions in the reconstruction [18].

compositional regions within a battery cathode in three dimensions. With careful sample preparation, APT has been found to be a very successful tool for associating concentrations of $\mathrm{Li}$ to the relative segregation of other elements as a function of battery charging and discharging [18]. The APT data of Figure $7(\mathrm{~g}-\mathrm{j})$ show the distributions of $\mathrm{Ni}, \mathrm{Mn}, \mathrm{O}$, and $\mathrm{Li}$, revealing that $\mathrm{Li}$ is concentrated in the Mn-rich part of the electrode. By comparing the APT-measured distribution of all elements in cathode materials before and after cycling, a detailed understanding of cycling-induced nanoscale compositional changes can be obtained: for example, Ni alters the Li distribution and likely impacts battery performance.

Optimizing electrode structure for performance Having learned that component segregation occurs during cathode synthesis and operation, it is useful to explore the options of using different synthesis approaches to minimize or avoid such segregation and to correlate such changes to battery properties. The performance of batteries made of $\mathrm{Li}_{1.2} \mathrm{Ni}_{0.2} \mathrm{Mn}_{0.6} \mathrm{O}_{2}$ cathode materials processed by different methods is shown in Figure $8 \mathrm{a}$. Note that the hydrothermal-produced material shows significantly less degradation in performance upon cycling compared to material produced in other ways. The STEM/EDS images in Figures $8 \mathrm{~b}, 8 \mathrm{c}$, and $8 \mathrm{~d}$ indicate minimum Ni segregation for the hydrothermal processed material while both sol-gel processed material and co-precipitation processed material have significant $\mathrm{Ni}$ segregation to the surface. Upon correlating the Ni segregation to battery performance, it shows that material without initial $\mathrm{Ni}$ segregation retains a high level of battery performance after more than 150 charge/discharge cycles [19].

NanoSIMS was also used to confirm the change in surface $\mathrm{Ni} / \mathrm{Mn}$ distribution because it provides information about chemical composition with good depth resolution. The NanoSIMS-determined elemental distributions in $\mathrm{Li}_{1.2} \mathrm{Ni}_{0.2} \mathrm{Mn}_{0.6} \mathrm{O}_{2}$ cathode materials fabricated by hydrothermal and sol-gel methods are shown in Figure 9, confirming the significant decrease in surface-segregated $\mathrm{Ni}$ for the hydrothermal process [19].

\section{Discussion}

In the past decade we have witnessed significant progress toward high sensitivity, high spatial resolution, and increased temporal resolution for many microscopy and surface-analysis instruments. The in-situ or operando operation of certain instruments can accelerate understanding, but usually data from such studies still need to be correlated with complementary methods employing conventional or ex-situ analysis and/or system modeling.

The need for multiple methods suggests several challenges and opportunities:

1. Integration of different instrument or analytical concepts on the same platform such that data may be acquired at the same site from several methods under in-situ or operando conditions. New combinations of such methods need to be designed without degrading the capabilities of the different methods involved. For example, microfabrication techniques and manipulations should make possible the integration of SIMS and STEM [20].

2. For some tools the integration of capabilities in a single instrument is not (yet) possible and may not be desirable. The creation of methods to enable easy and "spatially registered" transfers of samples in a controlled manner to different instruments is critical for some studies.

3. Application of multiple methods for different types of research may require expertise beyond that available in one research group or even one institution. The need for such expertise favors instrument centers and national user facilities and has implications for training of the next generation of researchers.

4. Multiple data streams and real-time data acquisition place increased demands on methods of data collection, storage, processing, and integration; further development in these areas would be helpful.

\section{Conclusion}

A combination of tools provided complementary information at a variety of spatial resolutions for addressing issues related to improvements in advanced batteries. No single method provided 


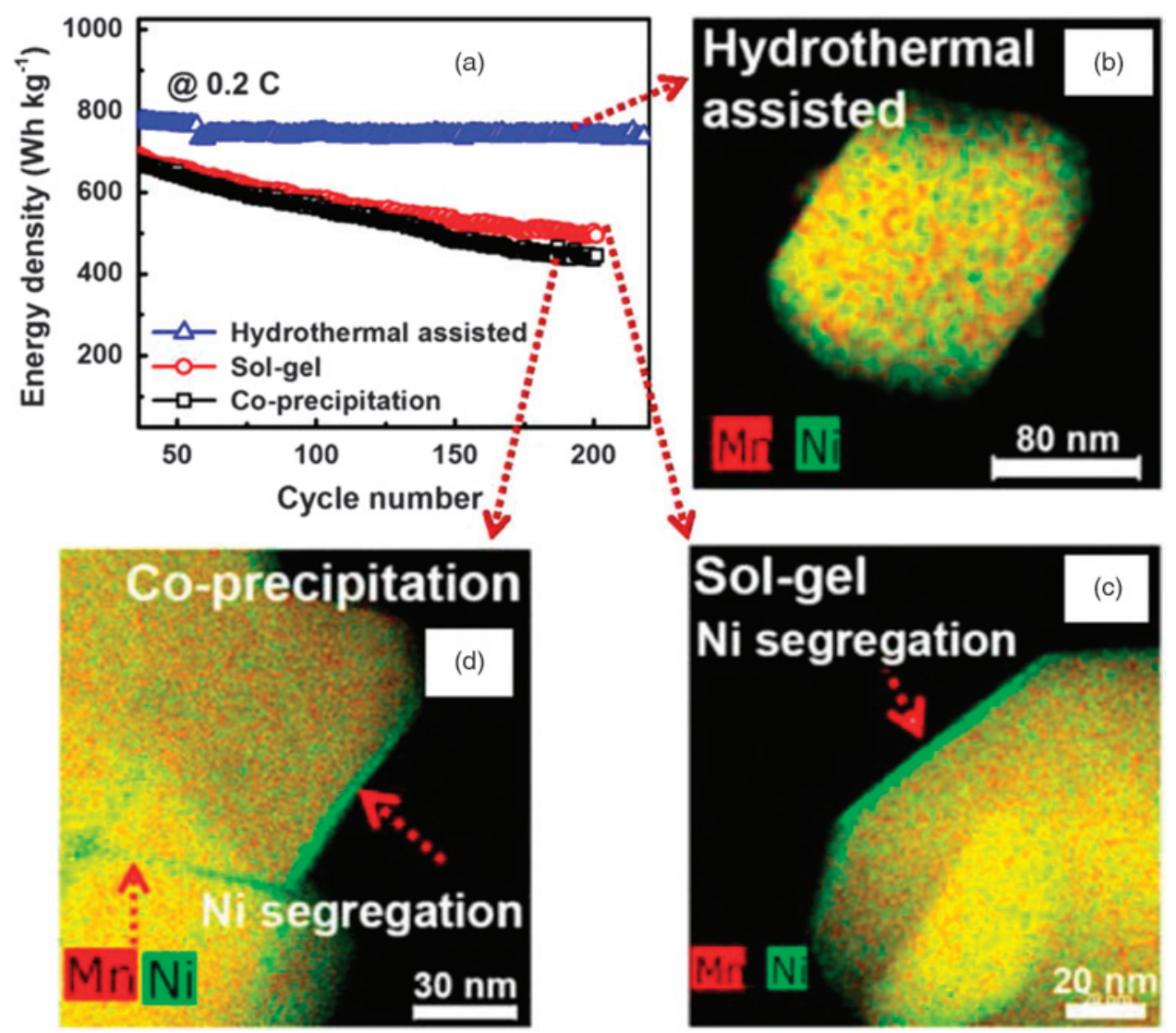

Figure 8: Correlation of structure and battery capacity retention for using $\mathrm{Li}_{1.2} \mathrm{Ni}_{0.2} \mathrm{Mn}_{0.6} \mathrm{O}_{2}$ as cathode for lithium battery. Note the $\mathrm{Ni}$ segregation is directly related to the processing route of the material, which directly affects the battery performance. (a) Energy density as a function of cycling number of the battery. (b) Hydrothermal-processed material showing minimum Ni segregation. (c) Sol-gel processed material shows Ni segregation. (d) Co-precipitation processed material shows $\mathrm{Ni}$ segregation.

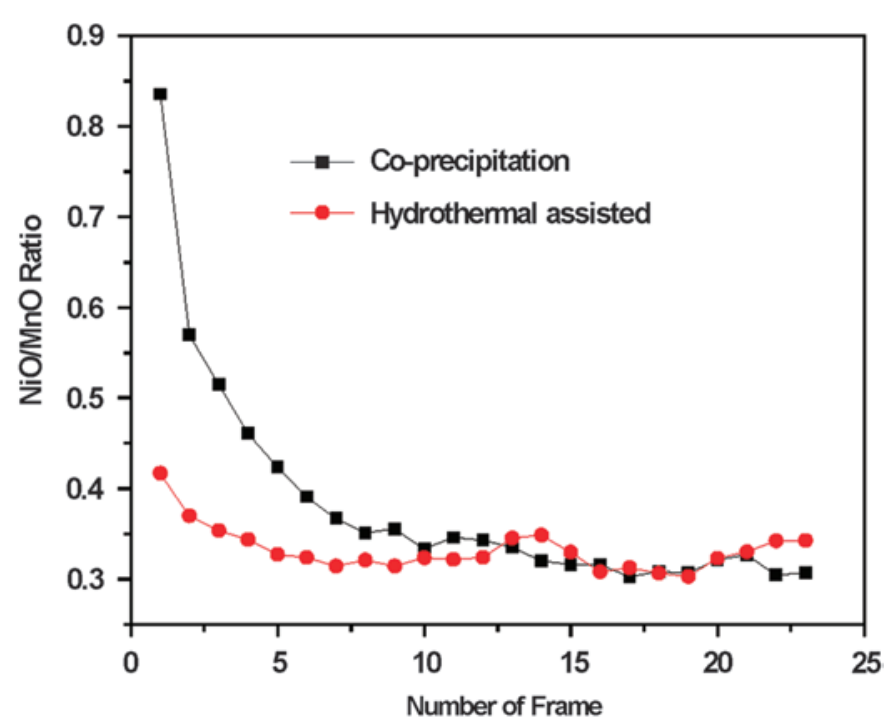

Figure 9: NanoSIMS depth profiles showing an enrichment of $\mathrm{NiO}$ at the surface of electrode material produced by co-precipitation and little or no $\mathrm{NiO}$ enrichment at the surface of hydrothermal grown materials. Each frame corresponds to 2 to $3 \mathrm{~nm}$.

the required range of information about elemental distributions, chemical states, and the evolution of structure and composition during charge/discharge cycles. The surface and interface sensitivity of XPS, different versions of SIMS, APT, and STEM

\section{References} 14141-51. (2015) 931. provide important information often difficult to obtain by other methods.

\section{Acknowledgements}

The topical work covered in this paper was enabled by the support of multiple programs centering on energy research including the Joint Center for Energy Storage Research, an Energy Innovation Hub funded by the Department of Energy (DOE), Office of Science, Basic Energy Sciences; the Assistant Secretary for Energy Efficiency and Renewable Energy, Office of Vehicle Technologies of DOE under Contract No. DE-AC02-05CH11231, Subcontract No. 6951379 under the advanced Battery Materials Research (BMR) program; and the Chemical Imaging Initiative, a Laboratory Directed Research and Development Program at Pacific Northwest National Laboratory (PNNL). Portions of the research were performed using the Environmental Molecular Sciences Laboratory, a national scientific user facility sponsored by the Department of Energy's Office of Biological and Environmental Research and located at PNNL. PNNL is a multiprogram national laboratory operated by Battelle for DOE under Contract DE-AC05- 76RL01830.

[1] JM Tarascon and M Armand, Nature 414 (2001) 359-67.

[2] M Armand and JM Tarascon, Nature 451 (2008) 652-57.

[3] P Yan et al., Nano Lett 15 (2015) 514-22.

[4] MT McDowell et al., Adv Mater 25 (2013) 4966-85.

[5] K Xu, Chem Rev 114 (2014) 11503-11618.

[6] PPRML Harks et al., J Power Sour 288 (2015) 92-105.

[7] CM Wang, J Mater Res 30 (2015) 326-39.

[8] H Zheng et al., MRS Bulletin 40 (2015) 12-18.

[9] CM Wang et al., J Mater Res 25 (2010) 1541-47.

[10] JY Huang et al., Science 330 (2010) 1515-20.

[11] M Gu et al., Nano Lett 13 (2013) 6106-12.

[12] BL Mehdi et al., Nano Lett 15 (2015) 2168-76.

[13] ZH Zhu et al., Nano Lett 15 (2015) 6170-76.

[14] EN Nasybulin et al., ACS Appl Mater Interf 6 (2014)

[15] MM Thackeray et al., J Mater Chem 15 (2005) 2257-67.

[16] M Gu et al., ACS Nano 7 (2013) 760-67.

[17] M Gu et al., Nano Lett 12 (2012) 5186-91.

[18] A Devaraj et al., Nature Commun 6 (2015) 8014.

[19] J Zheng et al., Nano Lett 14 (2014) 2628-35.

[20] S Eswara-Moorthy et al., Micros Microanal Suppl 2 


\section{MICROSCOPES}

\section{Isn't it about time you had your own Digital Microscope?}

Now you can with the portable, affordable uScopeMXII!

The uScopeMXII is a small digital desktop microscope you can use in your workplace or home office. It captures images from standard glass slides and sends them to your PC.

You can interactively browse slides with full control of focus, image processing, and location. You can also scan regions of interest creating fully focused image sets.

The industry-standard USB interface makes it simple to plug in and start capturing images. It easily interfaces with your desktop or laptop PC and allows you to view and capture slide images in a wide variety of environments.

The uScopeMXII is manufactured in the United States.

\section{Features and Benefits}

- Overview and Objective Cameras

The uScopeMXII has an objective camera for scanning and an overview camera for navigating.

- Automatic Focus Images are automatically focused using configurable focus algorithms.

- Portability

At a weight of about 5 lbs., the uScopeMXII is highly portable.

- Easy to Use

The uScope Navigator software simplifies scanning and browsing.

\section{- Self-Contained}

The uScopeMXII is self-contained and includes the electronics, cameras, stage, and optics in a device about the size of a large external disk drive.

\section{- Full Imaging Control}

User filters provide complete control over image processing and correction.

\section{Microscopes International, LLC}

555 Republic Drive, Suite 119

Plano, TX 75074-5498

$\begin{array}{ll}\text { Phone } & +1-214-785-2058 \\ \text { FAX } & +1-214-785-2138 \\ \text { Email } & \text { sales@uscopes.com } \\ & \text { support@uscopes.com }\end{array}$
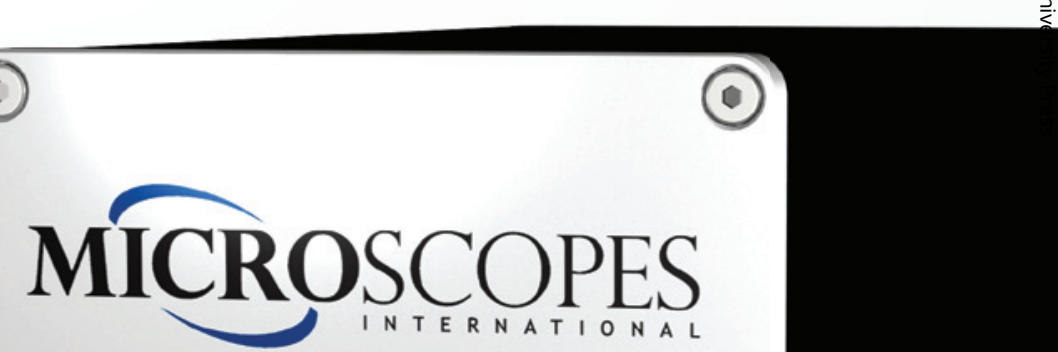

○)

uSCOPE" ${ }^{m \times \|}$

- POWER

- STATUS

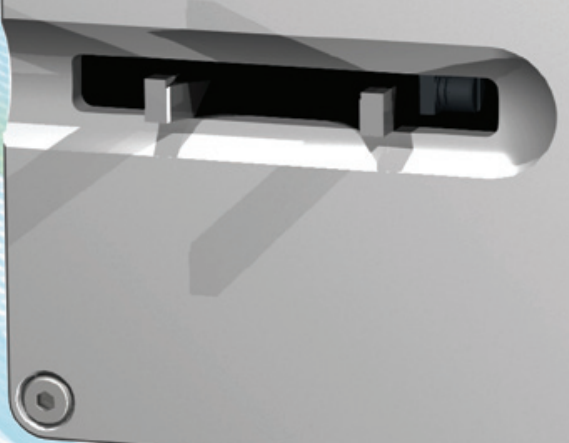
or to schedule a demonstration 